\title{
Verapamil is associated with an increased risk of cancer in the elderly: the Rotterdam study
}

\author{
A.B. Beiderbeck-Nolla,b, M.C.J.M. Sturkenboom ${ }^{\text {b }}$, P.D. van der Linden ${ }^{\mathrm{b}}$, \\ R.M.C. Herings ${ }^{\mathrm{a}}$, A. Hofman ${ }^{\mathrm{b}}$, J.W.W. Coebergh ${ }^{\mathrm{b}}$, H.G.M. Leufkens ${ }^{\mathrm{a}}$, \\ B.H.Ch. Strickerb,* \\ ${ }^{a}$ Department of Pharmacoepidemiology \& Pharmacotherapy, Utrecht Institute for Pharmaceutical Sciences (UIPS), Utrecht, The Netherlands \\ ${ }^{\mathrm{b}}$ Pharmaco-epidemiology Unit, Departments of Epidemiology \& Biostatistics and Internal Medicine, Erasmus Medical Center, \\ Rotterdam, The Netherlands
}

Received 10 December 2001; received in revised form 23 April 2002; accepted 15 May 2002

\begin{abstract}
The association between the use of calcium channel blockers (CCB) and cancer has received ample attention, but is still controversial. In this study, we have tested the hypothesis that the observed association between CCB and cancer in earlier studies could be explained by residual confounding or by misclassification of exposure because of the use of cross-sectional data on drug use. Data from the Rotterdam Study, a prospective population-based cohort study in the municipal area Ommoord, were used. The study population consisted of a cohort of 3204 participants aged 71 years or older who were followed from a baseline interview in the period 1991-1993 for the occurrence of incident cancer. Data on drug use were gathered at baseline and through the seven community pharmacies which served the Ommoord region during the study period between 1 January 1991 and 1 January 1999. Incident cancer events were gathered from a nationwide registry of hospitalisation data and from a specialised cancer centre in the Rotterdam region. We performed three analyses. First, we followed the method, and adjusted for the same risk factors, as in the earlier studies. In the second analysis, we included all risk factors that were univariately associated with cancer in the Rotterdam Study. In the third analysis, we included exposure to CCBs as time-varying co-variates, while adjusting for potential confounders. The relative risk (RR) of cancer associated with CCB was 1.4 (95\% Confidence Interval (CI): 0.9-2.0) in the first analysis and lowered to 1.2 (95\% CI: 0.8-1.8) upon adjustment for the different co-variates in the second. In both analyses, however, verapamil was significantly associated with cancer with RRs of 2.1 (95\% CI: 1.1-4.0) and 2.0 (1.01-3.9), respectively, whereas no associations were found with the other CCB in this study, i.e. diltiazem and nifedipine. A significantly increased risk of cancer was found for intermediate daily doses of verapamil and diltiazem. Intake of other antihypertensives such as $\beta$-blocking agents, diuretics and ACE-inhibitors was not associated with cancer. In the third analysis with exposure to CCB as time-varying co-variates, the risk increase was non-significant for use of 2 years or less, 1.0 (95\% CI: 0.7-1.5), and for use for a cumulative period of more than 2 years, 1.3 (95\% CI: 0.8-2.0). However, in all models the hazard ratio was statistically significantly increased for verapamil, but not for diltiazem and nifedipine. On the basis of these analyses, we found no increase in cancer in users of diltiazem and nifedipine, nor in users of other antihypertensives. In line with earlier studies, however, we found an increased risk of cancer in users of verapamil. At variance with the conclusions from several other studies, we think that it is too early to conclude that CCB are not associated with cancer.
\end{abstract}

(C) 2002 Elsevier Science Ltd. All rights reserved.

Keywords: Calcium channel blockers; Verapamil; Diltiazem; Nifedipine; Antihypertensives; Cancer; Adverse drug reactions; Pharmaco-epidemiology

\section{Introduction}

Calcium channel blockers (CCB) are widely prescribed for the treatment of hypertension and symptomatic

* Corresponding author. Tel.: + 31-10-408-7489; fax: + 31-10-4089382.

E-mail address: stricker@epib.fgg.eur.nl (B.H.C. Stricker). coronary artery disease. In 1996, concern arose about the association between long-term use of $\mathrm{CCB}$ in humans and cancer [1-7]. The hypothesised biological mechanism behind this suspicion was that $\mathrm{CCB}$ may inhibit the programmed death of premalignant cells by apoptosis, and may thereby promote tumour growth [4-7]. Others, however, have questioned this mechanism. The first epidemiological study on the association between 
$\mathrm{CCB}$ and cancer was published by Pahor and colleagues who performed a study in a cohort of 5052 people aged 71 years or more. Baseline data on drug exposure were collected in 1988 and cancer events were ascertained between 1988 and 1992. This first paper has led to an extensive correspondence that focused on potential selection bias, and misclassification of smoking and drug exposure. Since then, some epidemiological studies reported an increased risk $[5,6,8]$, while others did not demonstrate an association between CCB and cancer [9-15].

Because the first study made use of cross-sectional data on drug exposure, we anticipated that misclassification of exposure could be one of the reasons for a spurious association between $\mathrm{CCB}$ and cancer. Moreover, some potential confounders might not have been present in the first study, or might have been classified in a way that left room for residual confounding. Therefore, we tested our prior hypothesis that CCB are not associated with an increased risk of cancer. Hereto, we compared the analysis used in the initial study by Pahor and colleagues with two alternative approaches for the analysis of the association between CCB and cancer.

\section{Patients and methods}

\subsection{Setting}

The Rotterdam Study is a prospective populationbased cohort study of neurological, cardiovascular, locomotor and ophthalmological diseases in the elderly [16]. In brief, all inhabitants of Ommoord aged 55 years or older who were living in this municipal area of Rotterdam were invited in 1990-1993 to participate in the study. Of the 10275 eligible subjects, 7983 (78\%) participated and were interviewed at home. During this visit, trained interviewers administered an extensive questionnaire covering, among other topics, socio-economic background, food intake, medical history and current medication use (at day of interview). Baseline interviews were repeated in a second (1994-1996) and third (19971999) cross-sectional measurement. Additionally, as of 1991 all hospital admissions (coded according to the International Classification of Diseases (ICD)-9CM) of these patients are available in automated form. All patients obtain their prescribed medicines through seven pharmacies which serve the Ommoord region and surroundings, and which are fully automated and served by one common network. Over a 5-year-period, approximately $98 \%$ of the study population obtained at least one prescription through one of these pharmacies.

\subsection{Study cohort and period}

The potential study period consisted of the 8-year period between 1 January 1991 and 1 January 1999. We excluded all patients with a history of cancer before 1991. As we assumed that any tumour-inducing or promoting effect of calcium-antagonists would require an induction plus latent period of at least 1 year, we also excluded all patients who had a tumour which was diagnosed between 1 January 1991 and 1 January 1992 or who had filled a prescription for cytostatic agent before 1 January 1992. Furthermore, we excluded all participants below 71 years of age at study entry and all patients who were not registered with one of the pharmacies. The remaining population was followed from baseline until one of the following events: a registration of malignant cancer (ICD-9CM codes 140-208), death, transfer to another area, or end of the study period, whichever of the events occurred earliest.

\subsection{Exposure definition}

In order to study the effects of CCB on the estimated risk of cancer, we used two exposure definitions. In the first two analyses, we assessed exposure to CCB with data from the baseline interview in 1991, during which we collected information on all currently used drugs. At that time, patients were asked to show containers for all prescription drugs taken over the past 2 weeks before interview. In the third analysis, we applied time-varying cumulative exposure variables on the basis of pharmacy records over the entire study period as this facilitates adjustment for the duration of use as a risk factor. The pharmacy records contain automated information on all dispensed prescriptions since 1 January 1991 and include the product name, international non-proprietary name, the Anatomical Therapeutic Chemical (ATC) code (17), number of filled tablets/capsules or other dosage forms, the date of delivery, the prescribed daily number, the dosage and the legend duration (prescription length). For dosage comparisons, we expressed the prescribed daily dosages in defined daily doses (DDD). The DDD is a standardised dosing unit that was defined by the World Health Organization as the recommended daily dose of the drug for the main indication in an adult [17].

\subsection{Outcome definition}

Data on hospital admissions and discharge diagnoses for each subject were collected through a nationwide registry of hospitalisation data - which includes all admissions to all academic and general hospitals in the Rotterdam area - and from the Daniël den Hoed clinic which is a specialised cancer-referral centre in the Rotterdam region. For every admission, one mandatory main diagnosis and up to nine optional diagnoses are registered and stored in an automated database. As outcome, we used the first occurrence during the study period of a registration of malignant cancer (ICD-9CM codes 140-208). 


\subsection{Co-factors}

The following co-factors and risk indicators potentially related to the outcome cancer were collected at baseline: age, gender, hypertension (systolic blood pressure $\geqslant 160$ and/or diastolic blood pressure $\geqslant 95 \mathrm{mmHg}$, or the use of blood pressure lowering drugs with the indication hypertension), ischaemic heart disease (myocardial infarction verified by a cardiologist, or general practitioner and electrocardiogram (ECG), coronary bypass (number of coronary bypasses), PTCA, history of stroke (number of strokes), angina pectoris, peripheral artery disease, heart failure (defined as use of both, digoxin and a diuretic), diabetes mellitus, medication use (other antihypertensives, diuretics, $\beta$-blocking agents, ACE-inhibitors, corticosteroids for systemic use, antiinflammatory and antirheumatic products, sex hormones and modulators of the genital system, antilipidemics and HMG-CoA-reductase inhibitors), age at menopause, number of general practioner (GP) visits and hospital admissions before baseline, health complaints, socioeconomic status (highest education, current/last occupation), body mass index $\left(\mathrm{kg} / \mathrm{m}^{2}\right)$, cholesterol (total cholesterol and high density lipoprotein (HDL) cholesterol in mmol/l), smoking (in general, current smoking, ever smoked, past cigarette smoker, number of cigarettes currently smoked per day, number of cigarette packs per day multiplied by the total number of years of smoking for former smokers), alcohol ( $\geqslant 28.35 \mathrm{~g} /$ day, assessed by self-reported frequency). Moreover, we used the data from an extensive food questionnaire regarding the intake of energy, total fat, dietary fibre, vitamins C and $\mathrm{E}, \beta$-carotene and flavonoids.

\subsection{Analysis}

The aim of the study was to investigate whether inclusion of additional or better-defined potential confounders or a more detailed exposure assessment would take away the initially reported association between the use of CCBs and the occurrence of cancer. Therefore, we conducted three different analyses. The first analysis (model 1) exactly followed the methods of the initial study by Pahor and colleagues [4] and included the same potential confounders in the model. We assessed the rate of cancer in elderly ( $\geqslant 71$ years) users and nonusers of $\mathrm{CCB}$ at baseline by means of a Cox-proportional hazard regression model, while adjusting for age, gender, current smoking status, number of hospital admissions during follow-up, heart failure and alcohol intake. Since the prevalence of risk factors for cancer may differ between the Rotterdam study and the cohort that was used in the first study, we conducted a second analysis (model 2) in which we assessed the associations between all potential risk factors and the outcome cancer. All estimations were adjusted for age and gender.
Subsequently, we included in our second model all factors that were univariately associated with cancer $(P<0.10)$ and caused a change in the age- and gender-adjusted point estimate for exposure that was more than $5 \%$. In both analyses, we additionally conducted stratified analyses for individual $\mathrm{CCBs}$ and doses across different $\mathrm{CCB}$, ranking the dose as low (0.0-0.74 DDD), intermediate (0.75-1.24 DDD) and high (>1.24 DDD) [18]. In our third analysis (model 3, including all factors that were univariately associated with cancer $(P<0.10)$, diuretics, $\beta$-blocking agents and ACE-inhibitors), we calculated the relative risks of cancer with a Cox proportional hazards model in which exposure to CCB was obtained from pharmacy records and included as time-varying variables. As even chronically used drugs are often taken intermittently, we used dummy variables for the duration of use in these models. We constructed dummy variables for the duration of use since 1 January 1991, and distinguished exposure into two mutually exclusive groups of $\leqslant 2$ years and $>2$ years cumulative exposure, as the median of use in days was found to be close to 2 years. For Cox proportional hazard models, the assumption of proportionality was assessed by $\log$ minus log plots and by testing for interaction of exposure and co-variates with time, respectively. Adjustment for the effects of missing data was provided by additional indicator variables for almost all co-variates. For comparisons of proportions, we used $\chi^{2}$-statistics or a Fisher's exact test whenever the expected cell counts were less than 5. Comparisons between continuous data were performed with a Student's $t$-test or Mann-Whitney test in case of an abnormal distribution. All statistical tests were two-sided with a rejection of the null hypothesis at a $P$ value of $<0.05$.

\section{Results}

The original cohort consisted of 7983 elderly persons. From this population, we excluded 17 persons who were not registered with one of the pharmacies in the area of Ommoord, and 787 persons who died or had a history of cancer or use of cytostatics before 1 January 1992 or who had a follow-up (and thus medication history) of less than 6 months $(n=36)$. Furthermore, we excluded 69 persons with a diagnosis of cancer, but without further specification. The remaining study population comprised 7074 persons, of whom 29 had to be excluded because they had an index date before the study entry. Finally, similar to the study of Pahor and colleagues, we excluded 3841 individuals below 71 years of age leaving a cohort of 3204 participants for our analyses.

As expected, the 262 participants taking CCB were significantly more likely to suffer from cardiovascular diseases, diabetes mellitus and a history of stroke than the 2942 non-users. They also more frequently used 
antilipidemic agents, cardiovascular drugs and systemic corticosteroids. Finally, they reported more health complaints and had more hospital admissions. Several other potential risk factors did not differ significantly between the index and reference cohorts (Table 1). During 16640 person-years of follow-up (mean 5.2 years), 302 persons developed malignancies. The agespecific incidence rates per 100000 person years were 1437.7 for women and 2639.4 for men of 71-75 years, respectively (Table 2). Apparently, the cancer rate declined with advancing age. The most frequent cancers concerned the colon, prostate, skin, lung and a combination of the bladder, ureter and kidney (Table 3). In 29 users (mean follow-up time 4.7 years) and in 273 nonusers of CCB (mean follow-up time of 5.2 years) cancer was diagnosed (Table 4).

In our model 1, which was identical to that used in the study of Pahor and colleagues, the risk of cancer among $\mathrm{CCB}$ users was non-significantly increased by $36 \%$, after adjustment for age, gender, heart failure (defined as the use of diuretics and digoxin), current smoking status (number of cigarettes currently smoked per day), number of hospital admissions (before the qualifying event or before the end of follow-up) and alcohol intake $(\geqslant 28.35 \mathrm{~g} /$ day, assessed by self-reported frequency). In subsequent models, we used model 1 as a starting point,

Table 1

Characteristics of the study population at baseline $(n=3204)$

\begin{tabular}{|c|c|c|c|}
\hline Characteristic & Users of CCB $(\%)(n=262)$ & Non-users of CCB $(n=2942)$ & $P$ value \\
\hline Mean age (S.E.) & $79.2(0.37)$ & $79.3(0.12)$ & 0.76 \\
\hline Women & 64.5 & 67.9 & 0.26 \\
\hline Hypertension & 20.2 & 22.7 & 0.25 \\
\hline Ischaemic heart disease & 29.8 & 12.4 & $<0.001$ \\
\hline Coronary bypass ( $1-5$ events) & 4.6 & 1.8 & 0.004 \\
\hline PTCA ( $1-5$ events $)$ & 2.7 & 0.4 & 0.001 \\
\hline History of stroke & 7.3 & 5.4 & 0.25 \\
\hline Angina pectoris & 21.0 & 6.9 & $<0.001$ \\
\hline Peripheral artery disease & 26.7 & 22.0 & 0.12 \\
\hline Diabetes mellitus & 19.1 & 12.9 & 0.01 \\
\hline Heart failure & 11.1 & 4.9 & $<0.001$ \\
\hline \multicolumn{4}{|l|}{ Co-medication } \\
\hline Diuretics & 39.7 & 23.3 & $<0.001$ \\
\hline$\beta$-Blocking agents & 25.2 & 14.1 & $<0.001$ \\
\hline ACE inhibitors & 13.0 & 6.2 & $<0.001$ \\
\hline Antilipidemics (all) & 3.8 & 1.0 & 0.001 \\
\hline HMG-CoA-reductase-inhibitors & 3.4 & 0.7 & 0.001 \\
\hline Corticosteroids for systemic use & 5.0 & 2.4 & 0.01 \\
\hline \multicolumn{4}{|l|}{ Body mass index $\left(\mathrm{kg} / \mathrm{m}^{2}\right)$} \\
\hline$<20$ & 2.7 & 3.4 & Reference \\
\hline $20-25$ & 26.3 & 26.8 & 0.58 \\
\hline $25-30$ & 35.5 & 36.3 & 0.59 \\
\hline$>30$ & 17.9 & 12.4 & 0.14 \\
\hline \multicolumn{4}{|l|}{ Alcohol, self-reported } \\
\hline$\geqslant 28.35 \mathrm{~g} /$ day & 4.2 & 4.7 & 0.7 \\
\hline \multicolumn{4}{|l|}{ Current smoking } \\
\hline Non-smokers & 83.6 & 79.7 & Reference \\
\hline Smoking 1-19 cigarettes/day & 10.3 & 9.8 & 0.99 \\
\hline$\geqslant 20$ cigarettes/day & 1.5 & 2.7 & 0.23 \\
\hline \multicolumn{4}{|l|}{ Smoking (in general) } \\
\hline Current & 13.0 & 15.1 & Reference \\
\hline Former & 38.9 & 33.7 & 0.15 \\
\hline Never & 44.3 & 44.7 & 0.49 \\
\hline \multicolumn{4}{|l|}{ Hospital admissions } \\
\hline None & 59.9 & 61.1 & Reference \\
\hline 1 admission & 16.8 & 18.6 & 0.63 \\
\hline 2 admissions & 7.6 & 10.2 & 0.26 \\
\hline 3 admissions & 5.7 & 4.8 & 0.47 \\
\hline$\geqslant 4$ admissions & 9.9 & 5.3 & 0.004 \\
\hline \multicolumn{4}{|l|}{ Highest education } \\
\hline Primary school & 40.8 & 37.7 & Reference \\
\hline Lower vocational school/lower secondary school & 29.0 & 28.1 & 0.76 \\
\hline Intermediate vocational school/general secondary school & 19.5 & 20.1 & 0.54 \\
\hline Higher vocational school/university & 7.3 & 5.2 & 0.34 \\
\hline
\end{tabular}

S.E. standard error. 
Table 2

Age- specific incidence rates per 100000 person-years for cancer

\begin{tabular}{lll}
\hline Age at entry (years) & Incidence rates in women $(95 \% \mathrm{CI})$ & Incidence rates in men $(95 \% \mathrm{CI})$ \\
\hline $71-75$ & $1437.7(1092.4-1782.9)$ & $2639.4(2034.1-3244.7)$ \\
$76-80$ & $1075.0(717.9-1432.0)$ & $3597.8(2744.4-4451.3)$ \\
$81-85$ & $1738.6(1222.7-2254.5)$ & $2739.3(1645.4-3833.3)$ \\
$86-90$ & $1564.5(904.7-2224.3)$ & $1272.6(64.5-2480.7)$ \\
\hline
\end{tabular}

95\% CI, 95\% Confidence Intervals.

Table 3

Use of calcium channel blockers and their relationship with specific cancer sites

\begin{tabular}{lllll}
\hline Type of cancer (ICD-9 code) & Cancer events (\%) & Crude $^{\mathrm{a}}$ RR (95\% CI) & Model 1 $1^{\mathrm{a}}$ RR (95\% CI) & Model 2a RR (95\% CI) \\
\hline Stomach (151) & $11(3.6)$ & - & - & - \\
Colon (153) & $43(14.2)$ & $1.3(0.5-3.7)$ & $1.4(0.5-3.8)$ & $1.2(0.4-3.5)$ \\
Rectum (154) & $16(5.3)$ & $1.8(0.4-7.8)$ & $2.0(0.5-8.8)$ & $1.5(0.3-6.7)$ \\
Liver, gallbladder, pancreas (155-157) & $10(3.3)$ & $3.1(0.7-14.7)$ & $3.1(0.6-14.9)$ & $2.5(0.5-12.4)$ \\
Lung (162) & $24(7.9)$ & $1.0(0.2-4.4)$ & $1.3(0.3-5.5)$ & $0.8(0.2-3.5)$ \\
Skin (172-173) & $26(8.6)$ & $\mathbf{2 . 8 3 ( 1 . 1 - 7 . 5 )}$ & $\mathbf{2 . 7}(\mathbf{1 . 0 3}-7.3)$ & $2.4(0.8-6.6)$ \\
Breast (174) & $20\left(12.8^{\mathrm{b}}\right)$ & - & - & - \\
Uterus, adnexa (182-183) & $11\left(7.1^{\mathrm{b}}\right)$ & $1.4(0.2-10.8)$ & $1.8(0.2-13.8)$ & $1.1(0.1-9.0)$ \\
Prostate (185) & $32\left(21.9^{\mathrm{b}}\right)$ & $0.8(0.2-3.2)$ & $0.7(0.2-3.2)$ & $0.8(0.2-3.3)$ \\
Bladder, ureter, kidney (188-189) & $26(8.6)$ & $1.5(0.5-5.1)$ & $1.5(0.5-5.1)$ & $1.5(0.4-5.1)$ \\
Lymphatic, haematopoietic (200-208) & $15(5.0)$ & $1.8(0.4-8.3)$ & $2.0(0.4-8.9)$ & $2.0(0.4-9.0)$
\end{tabular}

Relative Risk.

${ }^{\text {a }}$ Crude RRs are adjusted for age and gender; model 1 is adjusted for age, gender, heart failure, current smoking status, number of hospital admissions as continuous and alcohol intake (according to Pahor and colleagues [4]); model 2 is adjusted for age, gender, ischaemic heart disease, total cholesterol $(\mathrm{mmol} / \mathrm{l})$, diabetes mellitus (forward stepwise multivariate analysis with variables which are univariately associated at a $P$ value $<0.1$ ).

b Percent of gender-specific cancer sites.

but included stepwise all factors which were associated with cancer in the Rotterdam cohort. An association with cancer $(P<0.10)$ was found for ischaemic heart disease, total cholesterol (mmol/l), diabetes (yes/no) and age at menopause in women, but not for any of the other factors, including those from the food questionnaire. No association with cancer $(P<0.10)$ was found for any of the smoking variables as given in the methods. Inclusion of the univariately associated factors in the multivariate analysis hardly changed the point estimate of the relative risk (RR) and the results are not shown here. In the analysis with the second model in which we only included potential risk factors which were univariately associated with cancer in the Rotterdam Study and other antihypertensives, we found a non-significantly increased RR of 1.2 (Table 4). In both models, there was no association between $\beta$-blocking agents, ACE inhibitors, and diuretics and cancer (Table 4). Adjustment of models 1 and 2 for concurrent use of these antihypertensives hardly changed the point estimates. In our study population, almost all users of CCB used either verapamil, diltiazem or nifedipine. In separate analyses with models 1 and 2, the association was significantly increased for verapamil, but not for diltiazem and nifedipine (Table 4). Further separate adjustment for use of ACE inhibitors, $\beta$-blocking agents and diuretics did not change the increased risk estimate for verapamil. When stratified by daily dosage, the increased risk of cancer was mainly found for intermediate daily doses (0.75-1.24 DDD) of verapamil with a RR of (2.7 (95\% Confidence Interval (CI): 1.02-7.4) while a daily dose below 0.75 DDD was associated with a non-significant RR of 1.7 (95\% CI: 0.7-4.2). A significant risk increase was also seen for intermediate doses of diltiazem with a RR of 2.1 (95\% CI: 1.05-4.3), but not for nifedipine. The high dosages for verapamil and diltiazem were not analysable because there were no cases in these strata (data not shown).

Because the cancer rates declined with age, we did a stratified analysis in two age groups: 71-79 years and $\geqslant 80$ years as was also performed in the study by Pahor and colleagues. The RR of cancer associated with the use of CCB in participants aged $71-79$ years $(n=1817)$ was 1.8 (95\% CI: $1.2-2.8)$ in this age stratum, but in participants aged 80 years and over the RR was 0.7 (95\% CI: $0.3-1.5)$. Further stratification according to 
Table 4

Use of antihypertensives and their association with cancer in the study population

\begin{tabular}{|c|c|c|c|c|}
\hline Antihypertensives & Cancer events & Crude $^{\mathrm{a}}$ RR $(95 \% \mathrm{CI})$ & Model $1^{\text {a }}$ RR $(95 \%$ CI $)$ & Model $2^{\mathrm{a}} \mathrm{RR}(95 \% \mathrm{CI})$ \\
\hline \multicolumn{5}{|l|}{$\beta$-Blocking agents } \\
\hline Users & 52 & $1.1(0.8-1.5)$ & $1.2(0.9-1.7)$ & $1.1(0.8-1.5)$ \\
\hline Non-users & 250 & Reference & - & - \\
\hline \multicolumn{5}{|l|}{ ACE inhibitors } \\
\hline Users & 19 & $1.0(0.6-1.6)$ & $1.0(0.6-1.6)$ & $0.9(0.6-1.4)$ \\
\hline Non-users & 283 & Reference & - & - \\
\hline \multicolumn{5}{|l|}{ Diuretics } \\
\hline Users & 69 & $1.2(0.9-1.5)$ & $1.3(0.9-1.8)$ & $1.2(0.9-1.5)$ \\
\hline Non-users & 233 & Reference & - & - \\
\hline \multicolumn{5}{|c|}{ Calcium antagonists } \\
\hline Users & 29 & $1.3(0.9-1.9)$ & $1.4(0.9-2.0)$ & $1.2(0.8-1.8)$ \\
\hline Non-users & 273 & Reference & - & - \\
\hline Verapamil & 9 & $1.9(0.96-3.6)$ & $2.1(1.1-4.0)$ & $2.0(1.01-3.9)$ \\
\hline Diltiazem & 10 & $1.4(0.8-2.7)$ & $1.5(0.8-2.9)$ & $1.3(0.7-2.4)$ \\
\hline Nifedipine & 9 & $1.00(0.5-1.9)$ & $0.9(0.5-1.8)$ & $0.8(0.4-1.6)$ \\
\hline
\end{tabular}

a Crude RRs are adjusted for age and gender; model 1 is adjusted for age, gender, heart failure, current smoking status, number of hospital admissions as continuous and alcohol intake (according to Pahor and colleagues [4]); model 2 is adjusted for age, gender, ischaemic heart disease, total cholesterol $(\mathrm{mmol} / \mathrm{l})$, diabetes mellitus (forward stepwise multivariate analysis with variables which are univariately associated at a $P$ value $<0.1)$ and additionally for calcium channel blockers, verapamil, diltiazem, nifedipin adjusted for diuretics, ACE-inhibitors and beta-blockers.

gender, diabetes mellitus, smoking, ischaemic heart disease, alcohol intake, cholesterol, or age at menopause in women gave largely similar results to the multivariate analyses and no effect modification was seen (data not shown). Individual cancer sites were analysed according to the ICD-9CM codes for malignant cancers (140-208). In the age- and gender-adjusted model and model 1, only the hazard ratios of skin cancer associated with the CCB were statistically significantly increased. No significant risk estimates were found for rectum, lung, prostate and the other cancer sites (Table 3). In general, the risk estimates differed from those found by Pahor and colleagues. The risk of specific cancers associated with verapamil use compared with other CCB was generally higher, but only statistically significantly increased for cancers of the lymphatic and haematopoietic organs (RR: 7.84, 95\% CI 1.66-37.0).

In the model with the analysis of cumulative exposure to CCB into two mutually exclusive groups of $\leqslant 2$ years and $>2$ years, we found non-significantly increased risks for exposure of less than 2 years and more than 2 years of cumulative exposure (Table 5). Additional adjustment of the crude analysis and model 3 for concurrent use of other antihypertensives (diuretics, betablocking agents and ACE-inhibitors) hardly changed the point estimates. However, a significantly increased risk of cancer was found for more than 2 years of cumulative exposure to verapamil. None of the hazard ratios to nifedipine, diltiazem and amlodipine, defined as time-varying cumulative exposure was statistically significant.

\section{Discussion}

In this study, we tested the hypothesis that CCB are not associated with an increased risk of cancer. We hypothesised that the results from an earlier study might be

Table 5

The association between CCB as time-varying exposures and cancer compared with non-use

\begin{tabular}{|c|c|c|}
\hline $\begin{array}{l}\text { Cumulative time } \\
\text { of exposure }\end{array}$ & $\begin{array}{l}\text { Crude analysis } \\
\text { RR }(95 \% \text { CI })\end{array}$ & $\begin{array}{l}\text { Model 3a } \\
\text { RR }(95 \% \text { CI) }\end{array}$ \\
\hline \multicolumn{3}{|l|}{ All CCB } \\
\hline$\leqslant 2$ years & $1.1(0.8-1.6)$ & $1.0(0.7-1.5)$ \\
\hline$>2$ years & $1.4(0.9-2.1)$ & $1.3(0.8-2.0)$ \\
\hline \multicolumn{3}{|l|}{ Verapamil } \\
\hline$\leqslant 2$ years & $1.5(0.9-2.5)$ & $1.4(0.8-2.5)$ \\
\hline$>2$ years & $2.4(1.2-5.0)$ & $2.4(1.2-4.9)$ \\
\hline \multicolumn{3}{|l|}{ Diltiazem } \\
\hline$\leqslant 2$ years & $1.1(0.7-1.9)$ & $1.0(0.6-1.7)$ \\
\hline$>2$ years & $0.7(0.3-2.0)$ & $0.7(0.2-1.8)$ \\
\hline \multicolumn{3}{|l|}{ Nifedipine } \\
\hline$\leqslant 2$ years & $0.9(0.5-1.6)$ & $0.8(0.4-1.5)$ \\
\hline$>2$ years & $1.4(0.8-2.7)$ & $1.3(0.7-2.5)$ \\
\hline \multicolumn{3}{|l|}{ Amlodipine } \\
\hline$\leqslant 2$ years & $0.4(0.1-1.7)$ & $0.4(0.1-1.6)$ \\
\hline$>2$ years & $0.7(0.1-5.3)$ & $0.7(0.1-5.3)$ \\
\hline
\end{tabular}

a Model 3: adjusted for age, gender, ischaemic heart disease, total cholesterol ( $\mathrm{mmol} / \mathrm{l})$, diabetes mellitus, diuretics, beta-blocking agents and ACE-inhibitors. 
explained by residual confounding or exposure misclassification. As the Rotterdam study has much information on potential confounders and drug exposure data on a daily basis for all cohort members, we expected that additional adjustment with these data would take away the association. In fact, we found results, which were largely similar to the study by Pahor and colleagues [4]. Although we found a lower risk estimate for any CCB, in our study verapamil was also associated with a 2 -fold and significant risk increase after adjustment for all known independent risk factors for cancer. This increase was dose-dependent. Furthermore, also in our study CCB were associated with an increased risk of cancer in the 71-79 years age group. At variance with the study by Pahor and colleagues, we did not find a risk increase in those aged 80 years and over. This might be explained by the fact that the incidence rate of cancer decreases in the very old, while their chance of receiving third-line treatment with CCB because of cardiovascular morbidity increases. Consequently, in this age group, the competing risk of cardiovascular events may reduce the relative contribution of cancer to mortality. In addition, in the analysis with time-varying exposure to verapamil, we observed a risk increase with increasing cumulative duration of therapy.

Our study has some potential limitations. First, like other epidemiological studies selection bias, information bias or confounding may have played a role. As the Rotterdam Study is a prospective population-based cohort study with a $78 \%$ participation rate, however, substantial selection bias is unlikely. The chance that we missed symptomatic cases of cancer is small, as cancer will eventually lead to a hospital admission or registration at a specialised cancer centre. Information bias is also unlikely as all data on drug use were gathered before the disease onset, and the pharmacy data are automatically stored without prior knowledge of any research hypotheses. Some misclassification of outcome may have occurred, but seems unlikely as most cancer is verified by extensive diagnostic procedures and classification of tumour pathology. In addition, the cancer incidences in our study are comparable to the age-specific incidence rates documented by the Netherlands Cancer Registry for 1996 that show an increase of cancer incidence in the age group 70-85 years followed by a decrease above 85 years for both men and women [19]. By excluding cases of cancer during the first year of the study, we reduced the chance that the onset of the malignancy preceded the use of CCB. In our models 1 and 2 , we deliberately analysed the data with cross-sectional drug data from interview to facilitate the comparison with the first study by Pahor and colleagues. The analyses with the use of continuous data from pharmacies in variables for cumulative exposure data, were in line with the finding that verapamil may be associated with an increased risk of cancer. Even so, the medical history of drug use in our study population may be too short to study the association between drugs and cancer, which may have a very long induction and latent period. In our analysis, we tried to adjust for confounding by adjustment for all known risk factors. Despite an extensive comparison between cases and non-cases, only a few variables were univariately associated with cancer. Unfortunately, we could not fully adjust for some dietary factors and some other factors because of missing values in up to $50 \%$ of the study population. Second, because of low numbers our study may suffer from a lack of precision. Some of the confidence intervals are wide. Another disadvantage of the low numbers was that it was difficult to study the association between CCB and individual tumour types.

Earlier studies on the association between CCB use and cancer have added different viewpoints on this issue. Some studies including three cohort studies [4-6] and one case-control study [8] found a significantly increased risk of cancer related to $\mathrm{CCB}$ use, primarily with the use of verapamil $[4,5,8]$. The risk estimate for diltiazem use was correspondingly increased, but nonsignificant $[4,5,9,13]$. On the other hand, five case-control studies $[10,11,13,15,20]$ and several cohort studies $[9,12,14,21-24]$ and an analysis of verapamil use within a randomised controlled trial [25] suggested no association between $\mathrm{CCBs}$ and cancer. However, a major limitation of most of these studies is the exposure assessment at baseline without detailed information on continuous drug use [5,6,8-12,14,21-23]. This may have led to a misclassification of exposure. In addition, confounder adjustment was found to be insufficient in most of studies mentioned $[8,22,24]$, also due to the limitations of registrations $[9,15]$. Continuous exposure to $\mathrm{CCB}$ with a drug prescription history of several years and detailed use pattern is needed to investigate the association with cancer and is provided in only a few studies [13,15,24].

The biological mechanism is basically unknown. Some authors have suggested that CCB may have a negative influence on apoptosis, programmed cell death, which has been under investigation regarding its role in oncogenesis [26]. Transmembrane calcium signals influence and cause cell differentiation and apoptosis [27,28]. Therefore, CCB are suspected to promote cancer growth by inhibiting apoptosis [29]. In addition, it has been suggested that $\mathrm{CCB}$, such as verapamil, might alter drug/xenobiotic accumulation in cells through the inhibition of the energy-dependent P-glycoprotein which has a physiological protective function by secreting various toxins and xenobiotics [30,31].

In conclusion, we were unable to confirm our prior hypothesis that $\mathrm{CCB}$ are not associated with cancer and that the observed association between $\mathrm{CCB}$ and cancer in the first study on this subject could be explained by methodological pitfalls. In particular, we confirmed the positive association with verapamil that has been found 
in several other studies. However, our numbers were too low to study the association with different tumour types. We think that it is too early to conclude that the association between $\mathrm{CCB}$ and cancer is spurious. In view of the widespread use of $\mathrm{CCB}$, we think that more detailed studies with larger numbers are required to shed more light on this issue.

\section{Acknowledgements}

The cooperation of all medical doctors and pharmacists in the Ommoord region is gratefully acknowledged.

\section{References}

1. Ad Hoc Subcommittee of the Liaison Committee of the World Health Organisation the International Society of Hypertension. Effects of calcium antagonists on the risks of coronary heart disease, cancer and bleeding. J Hypertens 1997, 15, 105-115.

2. Furberg CD, Psaty BM, Meyer JV. Nifedipine. Dose-related increase in mortality in patients with coronary heart disease. Circulation 1995, 92, 1326-1331.

3. Pahor M, Guralnik JM, Furberg CD, Carbonin P, Havlik R. Risk of gastrointestinal haemorrhage with calcium antagonists in hypertensive persons over 67 years old. Lancet 1996, 347, 10611065.

4. Pahor M, Guralnik JM, Ferrucci L, et al. Calcium-channel blockade and incidence of cancer in aged populations. Lancet 1996, 348, 493-497.

5. Pahor M, Guralnik JM, Salive ME, Corti MC, Carbonin P, Havlik RJ. Do calcium channel blockers increase the risk of cancer? Am J Hypertens 1996, 9, 695-699.

6. Fitzpatrick AL, Daling JR, Furberg CD, Kronmal RA, Weissfeld JL. Use of calcium channel blockers and breast carcinoma risk in postmenopausal women. Cancer 1997, 80, 1438-1447.

7. Daling JR. Calcium channel blockers and cancer: is an association biologically plausible? Am J Hypertens 1996, 9, 713-714.

8. Hardell LFM, Axelson O. Case-control study on colon cancer regarding previous diseases and drug intake. Int J Oncol 1996, 8, 439-444.

9. Olsen JH, Toft Sorensen HT, Friis S, et al. Cancer risk in users of calcium channel blockers. Hypertension 1997, 29, 1091-1094.

10. Rosenberg L, Rao RS, Palmer JR, et al. Calcium channel blockers and the risk of cancer. JAMA 1998, 279, 1000-1004.

11. Vezina RM, Lesko SM, Rosenberg L, Shapiro S. Calcium channel blocker use and the risk of prostate cancer. Am J Hypertens 1998, 11, 1420-1425.

12. Jonas M, Goldbourt U, Boyko V, Mandelzweig L, Behar S, Reicher Reiss H. Nifedipine and cancer mortality: ten-year follow-up of patients after acute myocardial infarction. Cardiovasc Drugs Ther 2607, 1998, 12 177-181.

13. Jick H, Jick S, Derby LE, Vasilakis C, Myers MW, Meier CR. Calcium-channel blockers and risk of cancer. Lancet 1997, 349, $525-528$.
14. Michels KB, Rosner BA, Walker AM, et al. Calcium channel blockers, cancer incidence, and cancer mortality in a cohort of U.S. women: The Nurses' Health Study. Cancer 1998, 83, 2003 2007.

15. Meier CR, Derby LE, Jick SS, Jick H. Angiotensin-converting enzyme inhibitors, calcium channel blockers, and breast cancer. Arch Intern Med 2000, 160, 349-353.

16. Hofman A, Grobbee DE, de Jong PT, van den Ouweland FA. Determinants of disease and disability in the elderly: the Rotterdam Elderly Study. Eur J Epidemiol 1991, 7, 403-422.

17. WHO. Anatomical Therapeutic Classification (ATC) Index: Including Defined Daily Doses (DDD) for Plain Substances. Oslo, World Health Organization, 1992.

18. Herings RM, Stricker BH, de Boer A, Bakker A, Sturmans F. Benzodiazepines and the risk of falling leading to femur fractures. Dosage more important than elimination half-life. Arch Intern Med 1995, 155, 1801-1807.

19. VIKC. Incidence of Cancer in the Netherlands. Vereniging van Integrale Kankercentra 1996. Eighth Report of the Netherlands Cancer Registry.

20. Vaughan TL, Farrow DC, Hansten PD, et al. Risk of esophageal and gastric adenocarcinomas in relation to use of calcium channel blockers, asthma drugs, and other medications that promote gastroesophageal reflux. Cancer Epidemiol Biomarkers Prev 1998, 7, 749-756.

21. Cohen HJ, Pieper CF, Hanlon JT, Wall WE, Burchett BM, Havlik RJ. Calcium channel blockers and cancer. Am J Med 2000, 108, 210-215.

22. Trenkwalder P, Hendricks P, Hense HW. Treatment with calcium antagonists does not increase the risk of fatal or non-fatal cancer in an elderly mid-European population: results from STEPHY II. Starnberg Study on Epidemiology of Parkinsonism and Hypertension in the Elderly. J Hypertens 1998, 16, 1113 1116.

23. Braun S, Boyko V, Behar S, et al. Calcium channel blocking agents and risk of cancer in patients with coronary heart disease. Benzafibrate Infarction Prevention (BIP) Study Research Group. J Am Coll Cardiol 1998, 31, 804-808.

24. Hole DJ, Gillis CR, McCallum IR, et al. Cancer risk of hypertensive patients taking calcium antagonists. J Hypertens 1998, 16, 119-124.

25. Sajadieh A, Storm HH, Hansen JF. Verapamil and risk of cancer in patients with coronary artery disease. DAVIT Study Group. Danish Verapamil Infarction Trial. Am J Cardiol 1999, 83, 1419 1422 (A9).

26. Stewart BW. Mechanisms of apoptosis: integration of genetic, biochemical, and cellular indicators. J Natl Cancer Inst 1994, 86, 1286-1296.

27. Whitfield JF. Calcium signals and cancer. Crit Rev Oncog 1992, 3, 55-90.

28. Trump BF, Berezesky IK. Calcium-mediated cell injury and cell death. Faseb J 1995, 9, 219-228.

29. Furberg CD, Pahor M, Psaty BM. The unnecessary controversy. Eur Heart J 1996, 17, 1142-1147.

30. Ford JM, Hait WN. Pharmacology of drugs that alter multidrug resistance in cancer. Pharmacol Rev 1990, 42, 155-199.

31. Ford JM. Experimental reversal of P-glycoprotein-mediated multidrug resistance by pharmacological chemosensitisers. Eur J Cancer 1996, 32A, 991-1001. 